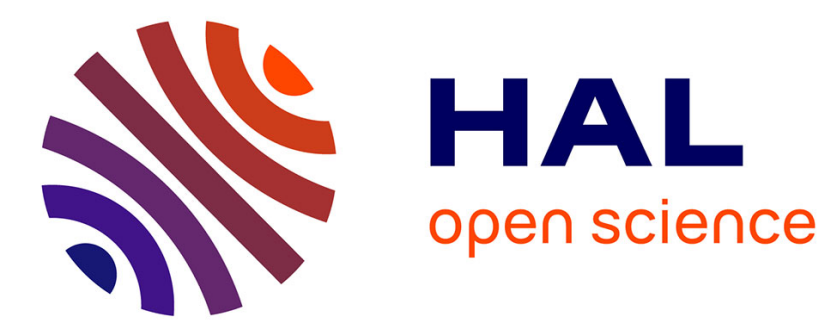

\title{
Mécanismes d'apprentissage sériel chez les vertébrés
} J.-F. Camps, V. Castéras, C. Mélan

\section{To cite this version:}

J.-F. Camps, V. Castéras, C. Mélan. Mécanismes d'apprentissage sériel chez les vertébrés. Annee Psychologique, 2002, 102 (4), pp.747-774. 10.3406/psy.2002.29618 . halshs-01612898

\section{HAL Id: halshs-01612898 \\ https://shs.hal.science/halshs-01612898}

Submitted on 8 Oct 2017

HAL is a multi-disciplinary open access archive for the deposit and dissemination of scientific research documents, whether they are published or not. The documents may come from teaching and research institutions in France or abroad, or from public or private research centers.
L'archive ouverte pluridisciplinaire HAL, est destinée au dépôt et à la diffusion de documents scientifiques de niveau recherche, publiés ou non, émanant des établissements d'enseignement et de recherche français ou étrangers, des laboratoires publics ou privés. 


\title{
J.-F. Camps \\ V. Castéras \\ C. Mélan
}

\section{Mécanismes d'apprentissage sériel chez les vertébrés}

\author{
In: L'année psychologique. 2002 vol. 102, n4. pp. 747-774.
}

\section{Résumé \\ Résumé}

Les mécanismes d'apprentissage sériel sont étudiés chez les vertébrés supérieurs grâce à deux types de protocoles. Dans l'un, l'animal doit reproduire l'ordre de présentation de plusieurs éléments présentés successivement, sollicitant fortement la mémoire à court terme. Dans l'autre, l'animal découvre l'ordre dans lequel il doit répondre à des éléments présentés simultanément. Les mécanismes d'apprentissage sériel incluent des processus discriminatifs, l'apprentissage de règles et le développement d'une représentation mentale. Ils ne s'excluent pas mutuellement pour un apprentissage donné, mais semblent varier selon l'espèce étudiée, et divers paramètres expérimentaux.

Mots-clés : apprentissage sériel, processus de discrimination, représentation mentale, comparaison interspécifique.

\section{Abstract}

Summary : Chaining processes underlying serial learning in vertebrates

Serial learning has been studied in vertebrate species using two different methods. One consists of the successive presentation of several items, and the animal has to respond according to this order. As shown by a systematic enhanced recall of the items presented last (recency effect), short-term memory processes appear to play a determinant role at least at the beginning of successive serial learning. The second method consists of the presentation of all items simultaneously, and the animal learns the correct order of responding. Serial learning mechanisms involve various discriminative processes (special status of the first and last items, conditional discrimination learning, temporal distance to the reinforcer), rule learning and the development of a mental representation (linear or associative) of the series. These mechanisms appear to vary according to the species tested, and according to several experimental parameters, such as the serial learning method, the learning procedure and the series' length. Involvement of more elaborate processes does not seem to rule out involvement of less elaborate processes at the beginning of training or on early learning phases with a partial series which precede later phases with the entire

Key words : serial learning, discriminative processes, mental representation, non-human species.

Citer ce document / Cite this document :

Camps J.-F., Castéras V., Mélan C. Mécanismes d'apprentissage sériel chez les vertébrés. In: L'année psychologique. 2002 vol. 102, n 4 . pp. 747-774.

doi : 10.3406/psy.2002.29618

http://www.persee.fr/web/revues/home/prescript/article/psy_0003-5033_2002_num_102_4_29618 


\title{
REVUE CRITIQUE
}

\author{
Laboratoire de Neurobiologie et Comportement \\ Université Toulouse 3, \\ Laboratoire Travail et Cognition* \\ Université Toulouse 2, CNRS CMR 5551'
}

\section{MÉCANISMES D'APPRENTISSAGE SÉRIEL CHEZ LES VERTÉBRÉS}

\author{
Jean-François CamPS, Vincent CaStéras \\ et Claudine MÉl.AN*.2.3
}

SUMMARY : Chaining processes underlying serial learning in vertebrates

Serial learning has been studied in vertebrate species using two different methods. One consists of the successive presentation of several items, and the animal has to respond according to this order. As shown by a systematic enhanced recall of the items presented last (recency effect), short-term memory processes appear to play a determinant role at least at the beginning of successive serial learning. The second method consists of the presentation of all items simultaneously, and the animal learns the correct order of responding. Serial learning mechanisms involve various discriminative processes (special status of the first and last items, conditional discrimination learning, temporal distance to the reinforcer), rule learning and the development of a mental representation (linear or associative) of the series. These mechanisms appear to vary according to the species tested, and according to several experimental parameters, such as the serial learning method, the learning procedure and the series' length. Involvement of more elaborate processes does not seem to rule out involvement of less elaborate processes at the beginning of training or on early learning phases with a partial series which precede later phases with the entire series.

Key words : serial learning, discriminative processes, mental representation, non-human species.

1. Maison de la Recherche, 5, allée Antonio-Machado, 31058 Toulouse Cedex 1.

2. E-mail : cmelan@univ-tlse2.fr.

3. Nous tenons à remercier Céline Lemercier pour ses recommandations sur la rédaction du document. 


\section{INTRODUCTION}

Ebbinghaus (1913) réalise les premières études expérimentales d'apprentissage sériel chez l'homme. Ses travaux ne susciteront l'intérêt des scientifiques qu'une cinquantaine d'années plus tard, lorsque Lashley (1951) met en avant l'importance de la mémoire de l'ordre sériel dans des activités élaborées telles que la compréhension et l'articulation du langage et la production musicale (pour une revue Lewandowsky et Murdock, 1989). La rédaction de cette phrase, par exemple, repose sur l'enchâ̂nement ordonné de lettres pour former des mots, puis sur l'enchaînement des mots selon certaines règles syntaxiques. Une multitude de travaux sont venus préciser les mécanismes d'apprentissage sériel chez l'homme dans des conditions expérimentales diverses, en faisant varier notamment la nature du matériel (verbal/non-verbal), les conditions d'apprentissage (implicite/explicite), les conditions de restitution (rappel/reconnaissance)...

L'objet de cette revue est de résumer l'état actuel de nos connaissances des mécanismes psychologiques d'apprentissage sériel chez l'animal. L'étude expérimentale des capacités d'apprentissage sériel chez l'animal se développe à partir des années 1970. Ces travaux visent à déterminer dans quelle mesure et de quelle manière un vertébré non doté de capacités symboliques apprend à mémoriser l'ordre d'informations arbitraires. Ils sont fondés sur l'observation que le comportement spontané de l'animal apparaît comme des séquences complexes d'actes ordonnés. Des études éthologiques ont révélé qu'un comportement adapté reflète l'intégration non seulement de plusieurs événements, mais également de l'ordre dans lequel ils se produisent (Camps et Mélan, 1999). Des séquences comportementales ritualisées caractérisent, par exemple, la parade sexuelle depuis les insectes jusqu'aux vertébrés supérieurs, en passant par les vertébrés inférieurs. À titre d'illustration, la parade sexuelle chez l'oiseau correspond à un enchâ̂nement précis d'orientations corporelles, de postures, de déplacements, de vocalisations et parfois de touchers entre mâle et femelle.

Dans un apprentissage sériel, l'animal apprend à répondre selon un ordre prédéterminé à plusieurs stimuli, appelés couramment items, qui sont présentés successivement ou simultanément (De Lillo, 1996). Les apprentissages sériels se distinguent d'autres types d'apprentissage essentiellement pour deux raisons : le nombre de stimuli et de réponses et l'ordre de ces réponses. Dans la majorité des apprentissages, l'animal est exposé à un seul ou à deux stimuli et on s'intéresse à la présence ou à l'absence de la seule réponse correcte. C'est le cas des épreuves de conditionnement classique et instrumental, tel que la discrimination entre deux compartiments ou allées. Grâce à des indices visuels (allée sombre / allée éclairée) ou spatiaux (allée gauche / allée droite) notamment, l'animal apprend à choisir le compartiment positivement renforcé ou à éviter le compartiment négativement renforcé. Dans un apprentissage sériel, en revanche, plusieurs stimuli 
sont présentés à chaque essai et l'animal doit émettre plusieurs réponses. Par exemple, des plages de couleurs $A, B, C$ et $D$ sont affichées sur des cibles et l'animal apprend à répondre aux quatre items. De plus, dans un apprentissage sériel l'animal apprend une séquence de réponses selon un ordre précis, d'abord l'item $A$, puis l'item $B$, puis $C$ et finalement $D$. Dans d'autres apprentissages fondés sur des réponses multiples, l'animal peut permuter au hasard la position de ses réponses dans la séquence. C'est le cas par exemple de l'épreuve d'élimination qui se déroule dans un labyrinthe radial. Celui-ci est constitué d'une plate-forme centrale d'où irradient huit allées ou plus. L'animal, placé sur la plate-forme centrale, apprend à parcourir chacune des allées une fois seulement. Il réalise alors bien une séquence de plusieurs réponses, mais l'ordre dans lequel il visite les différentes allées peut varier d'un essai à l'autre (Olton et Samuelson, 1976). L'originalité des apprentissages sériels tient alors à cette double spécificité : $1 /$ réponses multiples ; 2 / réalisées dans un ordre donné.

Cette spécificité de l'apprentissage sériel n'était pas reconnue au début du siècle. Ce point est développé dans une première partie au travers d'un bref rappel historique. La deuxième partie est consacrée à des tentatives de mise au point de dispositifs expérimentaux et de protocoles d'apprentissage spécifiques à l'étude de réponses multiples et ordonnées. La troisième partie montre que cet objectif a finalement été atteint dans les années 1970, grâce au développement de protocoles de parcours répétés d'une allée unique. Les deux parties suivantes sont consacrées aux travaux plus récents qui explorent l'apprentissage de séries lorsque différents stimuli sont présentés successivement ou simultanément. Dans le premier cas, l'animal apprend à reconnaître ou alors à reproduire un ordre donné de présentation, alors que, dans le second cas, il apprend à découvrir l'ordre dans lequel répondre aux stimuli. Les résultats expérimentaux obtenus dans ces travaux mettent en lumière le rôle que peuvent jouer des informations d'origine interne, telles que les manifestations proprioceptives corrélatives des réponses ou alors des images mentales des stimuli ou des réponses aux stimuli. L'identification et l'organisation de plusieurs réponses reposent également sur des informations d'origine externe, notamment la position particulièrement saillante de certains stimuli de la série. Une dernière partie montre que l'étude des mécanismes d'apprentissage sériel peut constituer un outil intéressant dans le domaine de la cognition comparée.

\section{RAPPEL HISTORIQUE}

Au début du siècle, des auteurs comme Watson (1913), Guthrie (1935) et Skinner (1938) postulent que tout apprentissage est le résultat de liens associatifs qui s'établissent entre un stimulus et une réponse. Thorndike (1913) propose que l'association entre un stimulus et une réponse dépend des conséquences de la réponse et de sa valeur fonctionnelle pour 
l'organisme. Selon les auteurs de ces théories, dites respectivement associationnistes et de renforcement, une séquence de réponses se réduit à un ensemble de réponses individuelles. Les dispositifs largement utilisés dans le domaine de l'apprentissage animal en général étaient également utilisés pour l'apprentissage de réponses multiples. Il s'agit de labyrinthes complexes, pourvus de plusieurs embranchements, par lesquels l'animal doit passer pour se déplacer d'un point donné jusqu'à un autre point où se trouve un renforcement alimentaire. Chaque embranchement correspond à un stimulus $(S)$ qui provoque une réponse conditionnée $(R ;$ modèle $S-R)$. Les conséquences proprioceptives engendrées par une réponse donnée participent comme stimulus à l'émission de la réponse suivante et ainsi de suite. Grâce au renforcement sélectif de la séquence correcte, des associations s'établiraient entre les réponses successives. De plus, suite à la distribution répétée du renforcement alimentaire immédiatement après une réponse au dernier item, une liaison conditionnée se formerait entre celui-ci et chacun des autres stimuli (Hull, 1931). En d'autres termes, l'idée d'une chaîne d'associations ou d'associations multiples serait suffisante pour expliquer l'apprentissage d'une séquence de réponses.

Lashley (1951) souligne que certaines observations sont réfractaires à une explication classique de chaînage successif. Il cite l'exemple des séquences comportementales formées de réponses successives qui sont très proches dans le temps. Parfois, les réponses sont trop rapprochées pour permettre aux stimulations proprioceptives qu'elles engendrent de devenir le stimulus de la réponse suivante. L'auteur inclut parmi ces séquences comportementales certains mouvements complexes chez l'animal et chez l'homme, mais également des activités humaines complexes, telles que la compréhension et la production du langage, ou la production musicale. À la même époque, Tolman (1948) postule que des associations se forment entre les stimuli eux-mêmes en fonction de buts précis, mais non pas entre des stimuli et des réponses. L'animal qui perçoit dans un labyrinthe un lieu particulier S1 (stimulus 1) s'attend à voir à proximité le lieu S2 (stimulus 2 ; modèle $S-S$ ). De telles associations par contiguïté spatiale s'étendraient ensuite et permettraient le développement d'une représentation mentale de l'espace labyrinthe. La théorie cognitive attribue un rôle particulier aux stimuli proches du renforcement : les lieux voisins de l'emplacement habituel de la nourriture sont affectés d'une attente particulière pour les animaux.

\section{LES TRAVAUX PIONNIERS}

Les premières théories d'apprentissage sériel étaient basées sur des observations réalisées dans des labyrinthes complexes. Or, dans cette situation expérimentale, les choix successifs de l'animal peuvent être guidés, en partie du moins, par des indices non liés directement à l'épreuve sérielle : 
des indices auditifs, olfactifs ou visuels qui sont intrinsèques ou extrinsèques au labyrinthe. Des dispositifs expérimentaux spécifiques à l'apprentissage sériel sont alors conçus afin de mettre formellement à l'épreuve les différentes théories.

\section{LE LABYRINTHE TEMPOREL}

Le premier dispositif correspond au labyrinthe temporel de Hunter (1920). Il est formé de deux allées, chacune en forme de boucle, qui se rejoignent au niveau d'une allée centrale. À partir d'un point de départ commun, l'animal doit faire un nombre défini de parcours dans une allée, puis dans l'autre allée. Pour apprendre une séquence formée de quatre parcours, dont les deux premiers sont réalisés dans une allée, et les deux suivants dans l'autre allée, l'animal doit se souvenir des deux derniers parcours afin de réaliser correctement le troisième, puis le quatrième parcours. Pour cela il ne peut se référer ni à des indices de l'environnement, ni aux manifestations proprioceptives de ses réponses puisque ces deux types d'informations restent inchangés entre parcours successifs dans la même allée. L'auteur postule que l'animal se réfère à des stimuli générés de manière interne et, plus précisément, à une représentation mentale de la séquence de parcours. Malgré son élégance, ce travail s'est heurté à un échec: des rongeurs n'ont pas pu apprendre une séquence formée de quatre parcours.

\section{APPRENTISSAGE D'UNE RËGLE D'ALTERNANCE}

Quarante ans plus tard, Bloom et Capaldi (1961) posent à nouveau la même question : l'animal est-il capable d'apprendre qu'une réponse donnée dépend des réponses précédentes? Ils s'inspirent des nombreux travaux qui montrent que des rongeurs apprennent à parcourir la même allée plus ou moins rapidement selon les conditions de renforcement. Dans un apprentissage de discrimination visuelle appétitive par exemple, des rongeurs apprennent à parcourir rapidement une allée en présence d'un indice visuel en vue d'un renforcement alimentaire. Ils parcourent la même allée lentement lorsque ce signal est absent. La différence du temps de parcours indique alors que les animaux apprennent bien la discrimination.

Dans le protocole modifié par Bloom et Capaldi (1961), aucun indice de discrimination n'est fourni mais une règle d'alternance simple est appliquée : le parcours de l'allée est successivement renforcé, puis non renforcé. Dans ce cas, des rats apprennent à effectuer un parcours lent suivant un essai renforcé et, inversement, un parcours rapide après un essai non renforcé. Ils effectuent alors bien une réponse donnée en fonction de la réponse précédente, mais en alternant deux réponses et non pas en réalisant des réponses multiples et ordonnées. Néanmoins, c'est grâce à ces travaux et en utilisant le même dispositif expérimental que le premier protocole d'apprentissage sériel a été développé. 


\section{SÉquences de PARCoURS DaNS UNE ALLÉE UNIQUE}

Dans les années 1970, Hulse et Campbell (1975) introduisent, finalement, la notion de série de stimuli. Dans un dispositif constitué d'une seule allée, l'animal récolte une quantité de nourriture différente lors de chacun des cinq parcours qui composent un essai. Des rats sont entraînés avec des séries monotones de nourriture, c'est-à-dire que le nombre de boulettes de nourriture augmente (0-1-3-7-14) ou diminue (14-7-3-1-0) de manière régulière à travers les parcours d'un essai, ou alors avec des séries aléatoires de nourriture (14-3-1-7-0). Dans le dernier cas, les séries varient à chaque essai. L'apprentissage est apprécié en comparant la vitesse de parcours de rats soumis à l'un ou à l'autre type de série.

1. APPRENTISSAGE IDANTICIPATION DE STIMUII CORRESPONDANT A UES QUANTITÉS VARIABLES DE NOURRITURE

Les résultats montrent que la vitesse de parcours des rats est proportionnelle à la quantité de nourriture récoltée pour les parcours des sêries monotones et constante pour les séries aléatoires. En d'autres termes, elle diminue entre les parcours successifs lorsque les quantités de nourriture diminuent également et, inversement, elle augmente pour des quantités croissantes de nourriture. Ces travaux montrent, pour la première fois, que des rongeurs sont capables d'apprendre une séquence ordonnée de réponses (Hulse et Campbell, 1975). Différentes explications théoriques sont proposées pour rendre compte de ces résultats.

\subsection{Anticipation à partir d'une règle formelle}

Selon Hulse (1978), les animaux apprennent la structure formelle d'une série qui leur permet d'anticiper le prochain choix selon la règle « plus grand que » pour une série croissante et «plus petit que " pour une série décroissante. À l'appui de cette hypothèse Hulse et Dorsky (1979) montrent que des animaux entraînés avec une première série monotone améliorent leurs performances lorsqu'ils apprennent ensuite une nouvelle série du même type. En revanche, l'acquisition d'une série aléatoire ne facilite pas l'apprentissage ultérieur d'une série monotone. En d'autres termes, le transfert est positif pour une série dont la structure est invariable alors que les quantités absolues de nourriture diffèrent, et négatif pour une série dont la structure change alors que les constituants restent inchangés. Les auteurs en concluent que les rats développent une représentation mentale d'une série au cours de l'apprentissage. 


\subsection{Anticipation par association des potentiels excitateurs relatifs des stimuli}

Capaldi, Verry et Davidson (1980) postulent qu'un potentiel excitateur relatif est associé à chaque item d'une série et que les stimuli adjacents s'associent par paires. Selon ce point de vue, l'évolution de la vitesse des parcours lors de l'apprentissage d'une série monotone de renforcements s'expliquerait par une évolution parallèle de la somme des potentiels excitateurs des stimuli. Les auteurs éprouvent cette hypothèse en présentant de manière aléatoire deux types de séries, comportant chacune cinq parcours. Le premier ou le dernier parcours est renforcé $(R)$ et les quatre autres sont non renforcés (N) $(\mathbf{R}-\mathbf{N}-\mathbf{N}-\mathbf{N}-\mathbf{N}$ et $\mathbf{N}-\mathbf{N}-\mathbf{N}-\mathbf{N}-\mathbf{R})$. À la fin de l'apprentissage, les rats parcourent lentement l'allée lors des quatre derniers parcours de la série commençant par un parcours renforcé. À l'inverse, ils parcourent de plus en plus rapidement l'allée lorsque c'est le dernier parcours de la série qui est renforcé (Capaldi et Verry, 1981). Ces résultats confirment alors l'idée que l'animal anticipe un renforcement « à venir 》 même si l'essai renforcé est précédé de plusieurs parcours non renforcés. Rien n'indique, cependant, qu'il ait intégré les parcours successifs sous forme d'une séquence, ni pour un type de série, ni pour l'autre.

\subsection{Apprentissage de propriétés plus globales d'une série}

Par la suite, Roitblat (1987) montre que la vitesse de parcours de rats renforcés par une série décroissante de nourriture (14-7-3-1-0) évolue de manière similaire lorsque un des items est ensuite remplacé par zéro boulette (indiqué en gras : 14-0-3-1-0). Puisque l'anticipation n'est pas affectée par l'intrusion occasionnelle d'un nouvel item, elle semble reposer sur des propriétés plus globales que la quantité de nourriture ou un potentiel excitateur. L'auteur propose que, dans cette situation expérimentale, les rats développent une représentation mentale de la séquence.

2. COMMENTAIRE : ÉVALLATION D'UN APPRENTISSAGE

A PARTIR IE IA VITESSE D'LNE RÉPONSE MOTRICE

En résumé, des interprétations très contrastées sont proposées pour rendre compte de l'apprentissage de parcours multiples d'une allée. Cette divergence tient en partie au fait que les quantités de nourriture correspondent à la fois aux stimuli qui composent une série et au renforcement des parcours correspondants. Il est difficile de dissocier le rôle joué par les items et les récompenses répétées ou, en d'autres termes, par la structure formelle et le potentiel excitateur. Par ailleurs, il se peut tout aussi bien que l'animal réalise chaque parcours par rapport à un stimulus et/ou un renforcement alimentaire donné. Dans ce cas il n'apprend pas nécessairement une 
séquence de réponses reliées entre elles par un ordre interne (Compton, 1991).

Un autre problème rencontré avec ce protocole concerne la mesure retenue pour évaluer les performances des animaux : la vitesse de parcours. La vitesse avec laquelle l'animal effectue une réponse motrice dans une situation d'apprentissage est en effet influencée par de nombreux facteurs. Des facteurs liés spécifiquement à un apprentissage, tels que les niveaux d'attention et de vigilance, influencent directement les performances motrices de l'animal. Des facteurs non spécifiques, notamment émotionnels et motivationnels, sont également susceptibles d'interférer avec la vitesse d'une réponse motrice. Celle-ci varie, par exemple, avec le niveau d'anxiété ou le degré de restriction alimentaire de l'animal (Mélan et Ungerer, 1991). Malgré ces réserves méthodologiques, les résultats qui sont issus de ces travaux constituent le fondement empirique de plusieurs hypothèses qui préfigurent les développements conceptuels ultérieurs.

\section{APPRENTISSAGES SÉRIELS SUCCESSIFS}

À partir des années 1980, les méthodes d'étude de l'apprentissage sériel changent de manière importante. Ces modifications concernent à la fois la nature des stimuli, le type de série et l'évaluation de l'apprentissage. Des stimuli non alimentaires sont utilisés, telles que des plages de couleur ou des images d'objets qui sont projetées sur des cibles. Ces stimuli forment des séries arbitraires, c'est-à-dire que leurs constituants ne sont pas reliés entre eux par un élément extérieur telle qu'une variation en taille ou en quantité. De plus, ils sont présentés d'emblée de sorte que l'apprentissage est évalué à partir des réponses de l'animal sur des cibles, et non plus à partir d'un paramètre moteur.

À partir de ces principes, deux types d'épreuves sérielles ont été développés : les apprentissages sériels successifs et simultanés. Cette section est consacrée aux protocoles d'apprentissages sériels successifs. Ils ont en commun la présentation des stimuli un par un sur une ou plusieurs cibles. L'animal doit discriminer un ordre donné de présentation ou alors le reproduire pour obtenir un renforcement alimentaire.

1. RECONNAISSANCE D'UN ORDRE DE PRESENTATION : DISCRIMINATION DE SÉQUENCES

La discrimination de séquences chez le pigeon est le protocole d'apprentissage sériel successif le plus utilisé. À chaque essai, l'animal est confronté à une séquence différente de stimuli, chacune correspondant à deux ou trois plages de couleur qui sont présentées successivement. Une seule séquence est correcte ou positive alors que toutes les autres séquences 
sont dites négatives : elles sont formées des mêmes stimuli que la séquence positive, mais qui sont présentés selon un ordre différent. La discrimination de la séquence positive des séquences négatives se traduit par une différence du taux des réponses de l'animal sur une cible neutre : il picore celleci lorsque c'est la séquence positive qui est présentée alors qu'il apprend progressivement à inhiber ses réponses sur cette cible en présence d'une des séquences négatives.

\section{I. Séquences de deux items : apprentissage de règles}

Lors de la discrimination d'une séquence positive de deux plages de couleur, $A$ puis $B$, les animaux discriminent d'abord les séquences à l'aide du second item : celles se terminant par l'item $B$ (AB, XB, BB ; Weisman, Wasserman, Nelson et Larew, 1980). Selon ces auteurs, une meilleure discrimination à partir de l'item présenté en dernier traduit un effet de récence. Les animaux discriminent ensuite la séquence positive $A B$ de la séquence négative $X B$, indiquant que le premier stimulus d'une séquence participe également à l'apprentissage discriminatif. Ce phénomène, désigné effet de primauté, est attribué à la saillance de l'item occupant la première position d'une série. On peut remarquer l'emploi inhabituel qui est fait des notions d'effet de récence et de primauté dans la littérature animale. Elles désignent ici simplement la supériorité de l'événement le plus récent ou de celui apparu en premier, alors qu'habituellement on emploie ces notions lorsque la série comporte au moins un élément intermédiaire. La discrimination de séquences à partir de règles basées sur la position des items est également observée par Wasserman, Nelson et Larew (1980). Ces auteurs montrent que l'amplitude de l'effet de récence diminue progressivement lorsque l'intervalle de rétention augmente de 0,5 à 2 , puis à 4 secondes. Un allongement comparable de l'intervalle de temps qui sépare la présentation des deux items de la séquence affecte peu ou pas les performances de rétention. En d'autres termes, après des délais de rétention courts l'animal accède à une séquence de deux items en mémoire à court terme. L'item présenté en dernier est cependant mieux mémorisé et semble de ce fait plus étroitement lié au renforcement.

\subsection{Séquences de trois items : formation d'une représentation mentale}

Plusieurs auteurs s'intéressent à la discrimination d'une séquence positive de trois items parmi une vingtaine de séquences négatives. Bever (1984) explore le rôle joué par les items occupant la première et la dernière position d'une séquence en supprimant certaines séquences négatives ou alors en utilisant plusieurs séquences positives. La discrimination de la séquence $\mathrm{ABC}$ s'installe plus rapidement lorsque les séquences négatives qui se terminent par l'item $C$ sont supprimées plutôt que celles débutant par l'item A. De même, les animaux discriminent plus facilement des séquences positives de longueur variable, lorsque celles-ci comportent 
l'item $C$ plutôt que l'item $A$ ( $A, A B$ et $A B C)$. L'auteur attribue le statut particulier du dernier item au fait qu'il précède immédiatement la réponse, ce qui le lierait en quelque sorte à celle-ci. Roitblat (1987) montre que les animaux qui ont appris à discriminer les séquences à partir de l'item $\mathrm{C}$ distinguent ensuite les séquences qui incluent à la fois l'item $\mathrm{A}$ et l'item $\mathrm{C}$. Par conséquent, les animaux semblent intégrer des combinaisons de stimuli en des unités fonctionnelles qui ne sont pas nécessairement organisées de manière séquentielle. En accord avec Terrace $(1986 a)$ et Bever (1984), cet auteur suggère que les animaux développent une représentation mentale de la séquence correcte qu'ils répéteraient mentalement à chaque essai pour $y$ comparer les stimuli présentés successivement.

\section{L'EPREUVE DE SHIMP : FORMATION D'UNITÉS FONCTIONNELLES}

Dans l'épreuve sérielle successive de Shimp (1976), l'animal doit indiquer la succession des positions qu'occupait un item présenté à plusieurs reprises. Deux cibles, disposées à gauche $(G)$ et à droite $(D)$ dans la boîte de conditionnement, permettent de présenter la séquence à apprendre : une croix blanche qui est projetée trois fois aléatoirement sur l'une ou l'autre cible. Huit séquences différentes sont utilisées, chacune correspondant à une succession différente de la croix sur les deux cibles (GGD, DDG, GDD, DGG, GDG, DGD, GGG, DDD). Après un intervalle de rétention, l'animal doit picorer les cibles dans le même ordre que celui utilisé lors de la présentation du stimulus. $\dot{A}$ cet effet, les deux cibles prennent successivement trois couleurs différentes, d'abord rouge, puis bleue et finalement blanche. L'animal doit picorer la cible sur laquelle la croix était présentée respectivement en première, en deuxième et en troisième position. Si les réponses successives de l'animal correspondent à la séquence de présentation, il est renforcé positivement après avoir effectué la dernière réponse.

Des pigeons apprennent à reproduire ainsi huit séquences différentes, tout en témoignant de performances plus élevées lorsqu'ils rappellent la position qu'occupait le stimulus en dernier. Lorsque l'intervalle de rétention est augmenté de 0,5 à 1 , puis à 4 secondes, les performances chutent globalement mais l'effet de récence persiste. Un allongement similaire du temps de présentation du premier item améliore la mémorisation de celuici, traduisant ainsi un effet de primauté. Shimp considère que la distribution répétée du renforcement après l'accomplissement de la séquence correcte permet d'expliquer la mémorisation des différentes séquences. L'obtention d'un renforcement favoriserait la consolidation en mémoire, non seulement du dernier item, mais d'une séquence dans sa totalité. Selon cette vue élargie de la théorie du renforcement, les items d'une séquence deviennent liés de manière fonctionnelle pour former ce que l'on appelle une unité fonctionnelle (ou chunk). 
3. APPARIENENT IOEXENPIAS PRESENTES SICCESSIVEMENT : APPRENTISSAGE DE SEQLENCES MLLTIPIES

L'épreuve d'appariement d'exemples présentés successivement (matching-to-successive samples) permet également d'étudier la mémorisation de l'ordre de présentation de stimuli chez l'animal. En début de chaque essai, deux ou trois stimuli (appelés « exemples ») sont présentés un à un, selon une séquence différente à chaque essai. Ensuite, ces stimuli apparaissent simultanément parmi d'autres stimuli ; tous sont alors appelés stimuli de comparaison. L'animal apprend à répondre uniquement aux stimuli de comparaison correspondant aux exemples présentés en début de l'essai, et cela selon leur ordre de présentation. Dans ce cas, l'essai se termine avec la distribution d'un renforcement alimentaire (renforcement positif). En revanche, une réponse selon un ordre incorrect ou aux stimuli de comparaison ne figurant pas dans la séquence correspond à une erreur. L'essai se termine alors et la cage de conditionnement est obscurcie pendant quelques secondes (renforcement négatif). On enregistre le taux de réponses correctes réalisées par séance d'apprentissage.

\subsection{Séquences multiples d'items issus de la même catégorie}

Des pigeons sont entraînés avec six séquences de deux exemples sélectionnés parmi trois plages de couleurs possibles. Un essai débute par la présentation de l'exemple $A$, puis de l'exemple $B$, qui apparaissent ensuite simultanément avec le stimulus $X$. Les animaux sont entraînés à répondre aux exemples selon l'ordre inverse de leur présentation initiale (B puis $A$ ) afin d'éviter un effet de récence (MacDonald, 1993). Les animaux apprennent rapidement à quel item répondre en premier (effet de récence), mais ils effectuent la seconde réponse au hasard, et ce même après 24 séances d'entraînement (environ 1700 essais). L'auteur poursuit alors l'entrấnement en supprimant le renforcement négatif qui suit habituellement cette erreur. Après 68 séances supplémentaires, soit plus de 14.000 essais au total, le taux de réponses correctes est supérieur au hasard pour cinq animaux sur huit. Lorsque la durée de présentation du premier exemple est allongée de 5 à 8 secondes ou celle du second exemple raccourcie de 5 à 2 secondes, les pigeons ont tendance à répondre d'abord au stimulus présenté en premier (effet de primauté). On retrouve ici les effets de récence et de primauté déjà décrits pour d'autres apprentissages sériels successifs (Shimp, 1976 ; Wasserman et al., 1980 ; Bever, 1984). Selon MacDonald, les résultats reflètent une discrimination de la force mnésique des deux stimuli, ou alors l'apprentissage d'une règle qui varie selon les contingences de renforcement. 


\subsection{Séquences multiples d'items issus de plusieurs catégories}

L'appariement de deux et de trois exemples a également été étudié en utilisant des items issus de plusieurs catégories différentes : plages de couleur, formes géométriques, barres parallèles. L'apprentissage de séquences de deux items se déroule en sept phases dont la complexité augmente en fonction de trois paramètres : le nombre d'exemples présentés (un seul, puis deux), le nombre de catégories dont sont issus les stimuli (de une à trois catégories) et le nombre de stimuli de comparaison (de deux jusqu'à huit) (Terrace, Chen et Jaswal, 1996). Les animaux maîtrisent l'appariement de deux exemples selon l'ordre de présentation (A puis B) en 33000 essais en moyenne.

L'entraînement se poursuit ensuite avec 162 séquences différentes de trois exemples. À chaque essai, les trois exemples sont présentés respectivement pendant 8,4 et 2 secondes, puis ils réapparaissent ensemble en tant que stimuli de comparaison. Les animaux commencent la majorité des essais par une réponse à l'item $C$ plutôt qu'à l'item $A$ et, dans les rares cas où ils répondent correctement à l'item $A$ d'abord, ils répondent ensuite à l'item $C$ dans $85 \%$ des cas. C'est pourquoi, après 90 séances d'entraînement, leur taux de réponses correctes ne dépasse pas le hasard. Les auteurs font remarquer qu'en raison de l'utilisation d'une séquence différente à chaque essai, les items qui occupent la première et la dernière position n'acquièrent pas la saillance habituellement observée pour ces items. Un deuxième groupe d'animaux est alors soumis à un préapprentissage dans une épreuve sérielle simultanée dans laquelle l'animal apprend à répondre selon un ordre prédéterminé et invariable à travers les essais, à trois stimuli présentés simultanément mais qui ne varient pas à chaque essai. Les animaux ainsi préentraînés apprennent ensuite la tâche d'appariement de trois exemples avec un taux de $90 \%$ de réponses correctes par séance. À partir des items occupant les positions les plus saillantes, les pigeons auraient formé des sous-unités de la série apprise au cours du préapprentissage, puis une image mentale de la série entière. Ce même mécanisme leur permettrait de mémoriser ensuite une séquence différente à chaque essai dans l'épreuve d'appariement d'exemples.

\section{DISCUSSION}

Les travaux résumés ici montrent que, au-delà de la reconnaissance ou de la discrimination d'un ordre de présentation de stimuli, l'animal est capable de reproduire cet ordre grâce à ses réponses successives. Indépendamment du protocole d'apprentissage employé, les auteurs établissent une distinction entre des séquences de deux items et de celles de trois items. L'apprentissage des premières est systématiquement expliqué par l'acquisition de règles et en particulier celle basée sur la plus grande saillance du second item. 
Lorsque les apprentissages portent sur des séquences de trois items, l'animal doit tenir compte de l'ordre des stimuli. Il développerait une représentation mentale de la séquence à discriminer ou à produire. À chaque essai, il comparerait les items présentés, stockés en mémoire de travail, à l'image mentale, conservée en mémoire de référence. L'hypothèse alternative d'une mémorisation par association des items d'une séquence paraît, au contraire, peu probable. En effet, la charge mnésique nécessaire à la mémorisation de tous les constituants des nombreuses séquences apprises dépasserait les capacités mnésiques du pigeon (Shimp, 1976 ; Terrace et al., 1996).

Dans l'ensemble des travaux rapportés ici, les résultats indiquent que le stimulus présenté en dernier est mémorisé plus rapidement ou mieux que celui ou ceux qui le précèdent. Par conséquent, les résultats s'expliquent partiellement par un traitement différentiel des stimuli en mémoire à court terme. C'est précisément en cela que les apprentissages sériels successifs se distinguent des apprentissages sériels simultanés qui sont décrits cidessous.

\section{APPRENTISSAGES SÉRIELS SIMULTANÉS}

Dans les apprentissages sériels simultanés, tels que leur nom l'indique, les stimuli sont présentés simultanément en début d'un essai. Ils ne se distinguent alors plus par leur récence relative. De plus, la configuration spatiale des items varie d'essai en essai, ce qui exclut la possibilité de se référer à des indices spatiaux. En 1979, Straub, Seidenberg, Bever et Terrace montrent, pour la première fois, que des pigeons apprennent l'ordre dans lequel répondre à quatre stimuli présentés en même temps, en l'absence de tout indice temporel ou spatial. Les mécanismes impliqués dans un apprentissage sériel simultané sont explorés grâce à différentes méthodes. Dans certains travaux, les animaux sont soumis à des tests après l'apprentissage d'une série, dans d'autres, on compare l'apprentissage de séries dont la composition diffère. Avant de résumer les résultats obtenus par des pigeons et des singes dans ce type d'apprentissage, nous allons brièvement décrire les caractéristiques communes à la plupart de ces protocoles expérimentaux.

\section{PROTOCOLE D'APPRENTISSAGE SERIEL SIMLLTANÉ : GÉḱkAlités}

Les résultats rapportés dans les paragraphes suivants sont obtenus dans les conditions expérimentales décrites ci-après (sauf si indiqué autrement). Dans les apprentissages sériels, un essai débute par la présentation simultanée de plusieurs stimuli visuels sur des cibles. En picorant les cibles et en appréciant les conséquences de ces réponses (renforcement alimentaire ou non) l'animal apprend à déduire l'ordre dans lequel il doit répondre 
pour être renforcé. La probabilité de répondre selon un ordre prédéterminé à plusieurs stimuli qui ne sont pas reliés entre eux par un indice extéroceptif temporel ou spatial, est cependant très faible. C'est pourquoi, un apprentissage sériel simultané est généralement établi progressivement, en plusieurs phases. Dans une première phase, l'animal est renforcé pour répondre à un seul stimulus (A) puis, dans une deuxième phase, il apprend à répondre dans l'ordre à deux stimuli, $A$ puis $B$, et ainsi de suite pour trois, quatre ou cinq stimuli.

Dans la plupart des travaux, seuls les stimuli à apprendre lors d'une phase d'apprentissage donnée sont présentés, c'est-à-dire les items A et $B$ lorsque l'animal doit apprendre la série AB. Le passage d'une phase d'apprentissage à la suivante se fait lorsque l'animal atteint un critère d'apprentissage, qui correspond généralement à $75 \%$ d'essais corrects par séance. Un essai est correct et l'animal est renforcé positivement après avoir répondu à l'ensemble des stimuli selon l'ordre à apprendre, tandis qu'il est incorrect et suivi d'un renforcement négatif (obscurcissement de la cage de conditionnement) après une réponse selon un ordre différent.

\section{EXPIORATION DES MÉCANISMES D'APPRENTISSAGE \\ À PARTIR d'ÉPRELVES POSTAPPRENTISSAGE}

Dans les travaux résumés ici, on évalue ce que l'animal a appris au cours d'un apprentissage sériel simultané grâce à divers tests réalisés après l'apprentissage. On s'intéresse aux performances des animaux lorsqu'ils sont confrontés à des sous-séquences de stimuli extraites de la série apprise ainsi qu'à l'apprentissage de nouvelles séries ou de nouvelles épreuves sérielles.

\section{1. Présentation de sous-séquences extraites d'une série apprise}

Cette méthode est employée par l'équipe de Terrace chez des pigeons ayant appris l'ordre des réponses à quatre ( $\mathrm{ABCD}$ ), voire cinq stimuli (ABCDE) (Straub et al., 1979 ; Straub et Terrace, 1981 ; Terrace, 1987, 1991). Confrontés ensuite à des paires et des triplets de stimuli extraits de ces séries, leur taux moyen de réponses correctes par séance est d'environ $80 \%$ pour toutes les sous-séquences confondues. Le taux des réponses correctes differe, cependant, entre les paires incluant le premier et/ou le dernier item de la série ( $\mathrm{AB}, \mathrm{AC}, \mathrm{AD} \ldots, \mathrm{AE}, \mathrm{BE}, \mathrm{CE} \ldots$ ), et celles formées des éléments intermédiaires de la série $(\mathrm{BC}, \mathrm{BD}, \mathrm{CD})$ : il est d'environ $90 \%$ pour les premières et de l'ordre de 50 à $60 \%$ pour les secondes.

Selon Straub et Terrace (1981), la mémorisation de sous-séquences de deux ou de trois stimuli peut s'expliquer par l'apprentissage de règles basées sur la saillance de certains items : « Répondre à l'item $A$ en premier, à l'item $E$ en dernier et à tout autre item par défaut. " En revanche, lorsque l'animal apprend une série plus longue, formée de quatre ou cinq 
stimuli, aucun indice discriminatif ne lui indique qu'après avoir picoré l'item B, par exemple, il doit répondre à l'item C plutôt qu'à l'item $A, D$ ou E. Les auteurs proposent que les pigeons forment une représentation mentale d'une série longue à laquelte ils se réfèrent pour réaliser les réponses successives aux différents items.

D'Amato (1991) propose, au contraire, que l'apprentissage d'une série de quatre ou cinq items s'explique par des mécanismes de conditionnement. Le choix de l'item A se ferait grâce à la discrimination de cet item en réponse à la présentation de la configuration de stimuli en début de l'essai. Les réponses aux items suivants de la série seraient basées sur le principe d'appariement conditionnel de l'exemple : après avoir picoré $A$, répondre à $B$ et ainsi de suite jusqu'à accomplissement de la série entière. Par ailleurs, D'Amato (1991) souligne que, face aux paires formées des éléments intérieurs de la série $\mathrm{ABCDE}$ (les paires $\mathrm{BC}, \mathrm{BD}, \mathrm{CD}$ ), les pigeons répondent dans la moitié des essais environ d'abord au deuxième item (items en gras). Il rapproche cette observation du fait que, lors de l'apprentissage de la série ABCDE, une réponse à ces items est plus proche de la distribution du renforcement alimentaire que celle au premier item de ces paires. Ces résultats indiqueraient que « des pigeons peuvent aussi apprendre quelque chose sur l'ordre temporel de la série d'items, dans le sens d'une discrimination de la distance temporelle relative qui sépare chaque item du renforcement» (D'Amato, 1991, p. 170)'. Selon D'Amato, la mise en jeu de ces divers mécanismes de conditionnement suggère tout au plus le développement d'une représentation faible de la série chez le pigeon.

Ces conclusions reposent également sur une comparaison des performances d'apprentissage sériel simultané du pigeon et du singe, entrainés dans des conditions expérimentales très proches ( $D^{\prime}$ Amato et Colombo, 1988). Des singes cébus présentent un taux de réponses correctes de $85 \%$ pour toutes les paires d'items issues de la série $\mathrm{ABCDE}$, indépendamment de la position des items dans la série. En d'autres termes, contrairement aux pigeons, les performances des singes sont élevées même pour les paires formées des éléments intérieurs de la série. De plus, la latence de leurs réponses au premier item d'une paire augmente proportionnellement avec sa position dans la série (AI < BI < CI..., I représente tout item apparaissant plus loin dans la série). Elle augmente également pour le second item d'une paire en fonction du nombre d'items qui le séparent du premier $(\mathrm{AB}<\mathrm{AC}<\mathrm{AD} \ldots)$. Ces données suggèrent que le singe développe une représentation linéaire de la série basée sur la connaissance ordinale de chaque item. Il passerait cette représentation mentalement en revue pour répondre dans l'ordre correct aux paires d'items, opération qui expliquerait que la latence de ses réponses varie en fonction des items composant une paire.

1. Pigeons may also learn something about the temporal ordering of the series items, in the sense of discriminating the relative temporal distance that separates each item from reinforcement. 


\subsection{Transfert à des séries dérivées de la série apprise}

L'objectif de ces travaux est d'évaluer la connaissance qu'acquièrent les animaux de la position des items d'une série. Des animaux apprennent d'abord la série $\mathrm{ABC}$, formée de plages de couleurs, puis des séries qui en sont dérivées en remplaçant un ou deux items par des items nouveaux, des formes géométriques ( $X$ et $Y$ ). Lorsque l'on remplace un item sans modifier la position des autres items, les séries dérivées sont du type $\mathrm{XBC}, \mathrm{AXC}$, $A B X, \ldots$; lorsque la position des autres items est modifiée, les séries dérivées sont du type XAB, BXA, BCX...

Dans cette tâche de transfert, les performances des pigeons chutent lorsque la position des items change entre l'apprentissage de la série initiale et celui des séries dérivées, indiquant un transfert négatif. Le transfert est positif, en revanche, lors du maintien de la position des items qui ne sont pas remplacés (Terrace, $1986 \mathrm{~b}$ ). Puisque les performances des pigeons sont affectées par une modification de la position des items de la série $A B C$, on peut penser qu'ils apprennent bien la position des constituants d'une série. Dans une étude ultérieure, des pigeons sont placés dans une épreuve de transfert portant sur des séries dérivées d'une série de quatre ou de cinq items. Ils répondent au hasard après 90 séances d'entraînement, sauf pour une série dérivée. Le transfert est en effet positif lorsque le nouvel item remplace le dernier item de la série apprise et que la position des autres items est maintenue (ABCX et ADCDX ; Terrace, Chen et Newman, 1995). Des singes entraînés avec des séries longues dans des conditions expérimentales similaires présentent, au contraire, des performances élevées indépendamment de la position qu'occupe le nouvel item dans les séries dérivées (D'Amato et Colombo, 1989).

Ces résultats confortent l'idée que les mécanismes d'apprentissage sériel different entre le singe et le pigeon. Chez le singe, l'apprentissage sériel aboutit à la connaissance de la position de tous les items d'une série même lorsque celle-ci comporte cinq items. Chez le pigeon, l'apprentissage de règles simples semble suffisant pour maîtriser une série courte et connaître la position des items qui la composent. Avec des séries longues, cette connaissance est limitée à l'item occupant la dernière position, plus facile à discriminer en raison de sa proximité temporelle avec la distribution du renforcement.

\subsection{Transfert à d'autres épreuves sérielles}

La maîtrise d'un apprentissage sériel simultané favorise également l'acquisition de certaines épreuves sérielles successives, décrites dans la partie précédente. La discrimination de la séquence positive $\mathrm{ABC}$ d'une vingtaine de séquences négatives est acquise en 53 séances en moyenne par des animaux naïfs et en $\mathbf{2 3}$ séances par des pigeons entraînés au préalable à produire la série ABC dans l'épreuve sérielle simultanée (Terrace, 1986 a). Une telle facilitation n'est pas observée chez des animaux témoins préentraînés à discriminer trois items individuellement et/ou à répondre à trois 
items qui ne constituent pas une série. De même, l’apprentissage de la série ABC dans un premier temps permet à des pigeons de maîtriser l'épreuve d'appariement de trois exemples dans un deuxième temps, alors que des animaux naïfs n'y parviennent pas (voir chapitre précédent, Terrace et al., 1996). À nouveau, des animaux préentraînés de la même manière que les témoins dans l'expérience de discrimination de séquences ne témoignent pas d'une facilitation dans l'épreuve d'appariement.

Les auteurs suggèrent que l'apprentissage sériel simultané aurait permis aux pigeons d'apprendre la position des trois items de la série et que cette connaissance ferait l'objet d'une représentation mentale. C'est en se référant à celle-ci qu'ils apprendraient ensuite avec plus de facilité un second apprentissage, également basé sur cette connaissance. Dans la tâche d'appariement, cette capacité de représenter une série permettrait au pigeon de mémoriser l'ordre des items utilisés à un essai donné afin de répondre correctement aux stimuli de comparaison.

\section{EXPLORATION À PARTIR DE L'APPRENTISSAGE DE SÉRIES DONT LES CARACTERISTIQUES VARIENT}

Certains auteurs ont étudié la manière dont l'animal organise ses réponses successives en une séquence ordonnée, en comparant les performances d'apprentissage d'animaux entraînés avec des séries dont la composition, la structure ou l'ordre d'enchaînement des items changent par rapport à ceux couramment employés dans le protocole d'apprentissage sériel simultané de base.

\subsection{Séries formées de sous-unités homogènes d'items et séries hétérogènes}

Des pigeons apprennent des séries qui sont composées uniquement de plages de couleurs (ABCDE) ou à la fois de plages de couleur et de formes géométriques (lettres accompagnées d'une apostrophe). Lorsque deux catégories d'items sont utilisées, les items sont regroupés par catégorie pour former deux sous-unités homogènes (ABCD'E'), ou alors ils sont dispersés pour former des séries hétérogènes ( $A B^{\prime} C^{\prime} D^{\prime} E$ ). Pour les animaux entraînés avec une série formée de deux sous-unités homogènes (ABCD'E'), le nombre de séances au critère d'apprentissage est diminué de moitié par rapport aux autres groupes d'animaux (Terrace, 1987, 1991). De plus, ils témoignent d'un taux de réponses correctes élevé face aux paires de stimuli extraites de la série apprise, même pour la paire CD', formée des items intermédiaires. Les animaux ayant appris la série chromatique ABCDE ou la série hétérogène $A B^{\prime} C D^{\prime} E$ répondent au hasard face à la paire équivalente, $C D$ et $C D$ ' respectivement. Ces résultats indiquent que les pigeons apprenant une série organisée en deux sous-unités homogènes auraient formé deux unités fonctionnelles (ABC et D'E'). Ceci ramènerait l'apprentissage d'une série longue à celui de deux séries plus courtes, impliquant 
une charge mnésique plus réduite et permettant une acquisition plus rapide. Dans ce cas, les items intermédiaires bénéficient du statut particulier d'éléments respectivement de fin et de début d'une sous-unité.

Dans une autre étude, des singes capucins apprennent d'abord à produire la série $\mathrm{ABC}$, formée d'items issus de trois catégories distinctes. Ils sont ensuite placés dans une épreuve de transfert dans laquelle plusieurs items de chaque catégorie sont présentés $\left(A^{1}, A^{2} \ldots, B^{1}, B^{2} \ldots, C^{1}, C^{2} \ldots\right)$. Lorsqu'ils sont libres d'organiser l'ordre de leurs réponses aux items d'une même catégorie $\left(A^{2} A^{1} A^{4} A^{3} B^{2} \ldots\right)$, ils apprennent à enchaîner jusqu'à douze items (De Lillo, 1996). Ces travaux montrent que singes et pigeons exploitent spontanément la possibilité d'organiser leurs réponses lorsque les séries à apprendre ne sont pas entièrement aléatoires. En revanche, lors de l'apprentissage de séries aléatoires, formées d'items entre lesquels n'existe aucun lien, ils ont recours à des mécanismes plus coûteux et moins efficaces. En particulier chez le pigeon, l'apprentissage de séries formées de quatre ou de cinq items pourrait dépasser leur empan mnésique. Ceci expliquerait la lenteur de l'apprentissage dans ces conditions et le faible taux de réponses correctes face aux paires formées des items intermédiaires de la série.

\subsection{Séries non structurées par la méthode des phases successives d'apprentissage}

Rappelons qu'un apprentissage sériel simultané se déroule généralement en plusieurs phases d'apprentissage. Lors des phases successives, seulement un, puis deux, trois et finalement quatre, voire cinq items sont présentés. Terrace (1993) fait remarquer qu'avec cette procédure d'apprentissage progressif la série est en quelque sorte structurée. Ainsi, une fois que l'animal a appris à répondre dans l'ordre aux items $A$ et $B$ par exemple, l'item $C$ est introduit à la phase d'apprentissage suivante. Il peut alors simplement reproduire la sous-séquence $A B$ apprise jusque-là et, en l'absence du renforcement habituel, répondre par défaut au nouvel item, l'item C. En d'autres termes, il peut baser ses réponses sur cette règle simple, « ajouter le nouvel item à la fin de la sous-séquence apprise ».

Ces considérations amènent l'auteur à s'intéresser alors au comportement instrumental de singes apprenant des séries qui ne sont pas structurées par la méthode d'apprentissage par phases. Les animaux apprennent au total quinze séries différentes formées de quatre items (photographies d'objets naturels). Ils sont entraînes aux huit premières séries selon la méthode habituelle de structuration d'une série grâce à l'ajout d'un nouvel item à chaque phase d'apprentissage. Les séries suivantes sont peu ou pas structurées : à partir de l'apprentissage de la neuvième série, trois items sont présentés simultanément dès le début de la tâche et, à partir de la quatorzième série, l'apprentissage débute par la présentation de quatre items (Swartz, Chen et Terrace, 1991). Le passage de séries structurées à des séries non structurées entraîne une baisse initiale des performances, puis une amélioration qui est attribuée au développement de deux stratégies 
complémentaires. Premièrement, « lorsque l'apprentissage commence avec la présentation simultanée de trois items ou plus (série non structurée), il est nécessaire de tester les conséquences des réponses à chaque item afin de déterminer sa position ordinale » (Terrace, 1993, p. 167)' ${ }^{1}$. Deuxièmement, une fois que le singe a identifié l'item $A$, puis l'item $B$, il les regrouperait en une unité fonctionnelle. Il identifierait ensuite l'item $C$ et l'intégrerait pour former l'unité ABC. Finalement, le seul item restant, l'item $D$, peut être ajouté par défaut à la fin de la série. En résumé, face à des séries non structurées, les singes développent et testent des hypothèses concernant les items et leur position, ensuite ils assemblent les items adjacents en des unités fonctionnelles.

\subsection{Apprentissage de séries par ajout des items en début ou en fin de série}

Dans l'ensemble des travaux décrits jusqu'ici, l'animal apprend à ajouter à chaque phase d'apprentissage un nouvel item à la fin de la sousséquence apprise jusque-là. Le nouvel item occupe alors toujours la position affectée d'une importance particulière au vu de sa proximité avec la distribution du renforcement. Ceci soulève la possibilité que l'apprentissage repose partiellement sur cet arrangement; hypothèse qui a été éprouvée en étudiant l'ajout d'un item en début de la sous-séquence apprise.

Des pigeons sont entraînés à produire la séquence formée des items $A$ et $B$ selon l'ordre inverse pour apprendre la série $\mathbf{B A}$. Ils doivent répondre en premier au nouvel item $B$, puis seulement à l'item $A$, appris lors de la première phase de l'apprentissage. Les pigeons persistent cependant à répondre à l'item A lors de la deuxième phase de l'apprentissage. Cette erreur termine à chaque fois l'essai et déclenche un renforcement négatif (obscurcissement de la cage expérimentale). La persévération de cette erreur aboutit finalement à une extinction des réponses instrumentales des animaux (Straub et Terrace, 1981). Nous avons alors appliqué la méthode dite de libre correction des erreurs avec laquelle les erreurs n'ont pas de conséquences sur le déroulement de l'essai. En dépit d'erreurs répétées, les stimuli restent affichés jusqu'à ce que l'animal réalise la séquence demandée. Alors seulement l'essai se termine et l'animal est renforcé. Quatre items $A, B, C$ et $D$ sont présentés dès le début de l'apprentissage, ce qui modifie les conditions d'apprentissage de deux manières. Les items $A$ et $B$ ne se distinguent alors plus par leur degré de familiarité et la présence des items distracteurs $\mathbf{C}$ et $\mathbf{D}$ réduit les contraintes de choix engendrées habituellement par la présentation des deux seuls items à apprendre. Les pigeons apprennent la série BA en un nombre de séances comparable à celui des animaux apprenant la série selon l'ordre habituel (série AB ; Mélan, Castéras, Corbière et Gallo, 1996 ;

1. If training begins with the simultaneous display of three or more items... it is necessary to test the consequences of responding to each item in order to determine its ordinal position. 
Camps, Durif, Mélan et Delius, 1998). L'analyse du taux des réponses sur les différents items révèle que tous les animaux distinguent les items $A$ et $B$ des items distracteurs dès la première séance d'apprentissage et répondent ensuite préférentiellement au second item de la série. Cette préférence est transitoire chez les animaux apprenant la série $A B$, alors qu'elle se maintient à travers les séances chez les animaux du groupe BA chez lesquels elle entraîne un nombre d'erreurs par essai supérieur. En d'autres termes, les animaux apprennent bien une série de deux items selon l'ordre inverse, mais ils témoignent d'un comportement instrumental moins efficace.

Les animaux sont ensuite entraînés à ajouter l'item $C$ au début (série CBA) ou à la fin (série $\mathrm{ABC}$ ) de la série apprise jusque-là. Une étude préliminaire portant sur l'apprentissage de la série $\mathrm{ABC}$ en situation de libre correction des erreurs a révélé que les animaux produisent de moins en moins la sous-séquence $A B$ qu'ils avaient pourtant apprise durant la phase d'apprentissage précédente. Malgré cela, la production de la sousséquence $\mathrm{AB}$ est positivement corrélée à celle de la série $\mathrm{ABC}$, indiquant qu'à chaque fois que les animaux produisent la sous-séquence $A B$ ils $y$ ajoutent l'item C dans $95 \%$ des cas (Castéras, 1996 ; Castéras, Camps et Mélan, 1997). Ces résultats suggèrent une difficulté de mémorisation de la sous-séquence apprise et/ou une perte de sa saillance lors de l'ajout d'un troisième item. Cette hypothèse est éprouvée en amenant la moitié des animaux des deux groupes à rappeler à chaque séance d'apprentissage d'abord la sous-séquence apprise précédemment, c'est-à-dire AB ou BA, avant de les entraîner à ajouter l'item $C$ à la fín (groupe $A B-A B C$ ) ou au début (groupe BA-CBA; Camps, 1998).

Les animaux du groupe AB-ABC apprennent la série deux fois plus rapidement que ceux du groupe $\mathrm{ABC}$ ( 30 vs 56 séances). Dans les deux groupes, les sous-séquences $\mathrm{AB}$ et $\mathrm{BC}$ sont produites à des taux significativement supérieurs au hasard, mais qui sont plus élevés pour les animaux du groupe AB-ABC. L'apprentissage de la série selon l'ordre inverse (groupe CBA) est également maîtrisé rapidement (4.2 séances), mais ces animaux produisent seulement la seconde transition à un taux supérieur au hasard et témoignent d'un nombre d'erreurs et de répétitions par essai supérieur aux autres groupes. Globalement, les animaux des trois groupes répondent le plus souvent à l'item qui occupe la dernière position de la série. Dans le dernier groupe (groupe BA-CBA), les animaux rappellent correctement la sous-séquence $B A$, mais ils produisent la série de trois items au mieux une fois par séance, ce qui conduit à l'extinction de leurs réponses instrumentales (Camps, Castéras, Mélan et Delius, 1998). Les résultats indiquent, en accord avec l'hypothèse de $D^{\prime}$ 'Amato (1991), que la facilité avec laquelle les animaux ajoutent un item dépend de la position de celui-ci dans la série. Situé en fín de série il est facile à discriminer au vu de sa proximité avec la distribution du renforcement (groupes ABC et ABABC), alors qu'en début de la série il est au contraire difficile à discriminer (groupes CBA et CB-CBA). La procédure de rappel permet de maintenir en mémoire une sous-séquence apprise, mais ses effets sur l'apprentissage 
dépendent à nouveau de la position de la sous-séquence dans la série de trois items : ils sont positifs si la sous-séquence rappelée se situe en début de la série et négatifs si elle se situe à la fin de la série. La supériorité très nette des performances des animaux du groupe AB-ABC peut alors s'expliquer par un effet double : 1 / la procédure de rappel leur permet de former une unité fonctionnelle des items situés en début de la série ; 2 / ils agrandissent cette unité en y ajoutant le troisième item dans la position la plus facile à discriminer (Shimp, 1976).

En situation de libre correction des erreurs, les performances d'apprentissage sériel simultané s'expliquent alors partiellement par les contingences de renforcement. Des expériences complémentaires montrent que cet effet est réduit lors d'un entraînement intensif. Des pigeons sont d'abord entraînés à produire la série BA parmi les quatre plages de couleurs $(A, B, C, D)$, puis la série $B^{\prime} A^{\prime}$ à partir d'autres plages de couleurs (A', B', $\left.C^{\prime}, D^{\prime}\right)$. Après le rappel des deux séries, les items sont mélangés et les animaux apprennent les séries $A B^{\prime}$ et $A^{\prime} B$ parmi les items respectivement $A B^{\prime} C^{\prime} D^{\prime}$ et $A^{\prime} B C^{\prime} D$. Ils apprennent les séries dérivées en un minimum de séances et répondent préférentiellement aux items dans l'ordre demandé et non plus en fonction de leur proximité avec le renforcement, indiquant qu'ils ont acquis une certaine connaissance de la position des items (Mélan, Galy et Camps, 1998).

\section{DISCUSSION}

Ces travaux montrent que, en l'absence de toute indication de l'ordre, le pigeon parvient à déduire celui-ci, en basant ses réponses sur différents types d'informations: distribution du renforcement, continuation/interruption d'un essai, structure de la série. À partir de ces indications il apprend la position des items qui composent une série. Grâce à un empan mnésique plus grand que les pigeons, les singes seraient capables de former une représentation mentale élaborée d'une série longue. Par ailleurs, les pigeons ont tendance à répéter spontanément une réponse qui a été renforcée, ce qui est à l'origine de comportements stéréoty pés. Ceci est particulièrement gênant dans des apprentissages sériels, puisque l'animal doit produire plusieurs réponses. La plus grande flexibilité comportementale des singes assure, au contraire, une adaptation plus facile aux contraintes d'une situation expérimentale.

\section{PERSPECTIVES ÉVOLUTIVES}

Les parties qui précèdent ont permis de montrer que les mécanismes d'apprentissage sériel sont étudiés chez plusieurs espèces de vertébrés supérieurs. À notre connaissance, des études similaires n'ont pas été réali- 
sées chez des vertébrés inférieurs. En revanche, quelques résultats intéressants ont été obtenus chez des insectes, et plus particulièrement chez des hyménoptères. Des fourmis Cataglyphis cursor sont amenées à retourner au nid depuis une source de nourriture en passant par un dispositif composé de quatre boîtes $(A, B, C, D)$ disposées en série. Chaque boîte possède deux orifices différenciés par un indice visuel signalant respectivement une voie sans issue $(A-)$ et l'accès à la boîte suivante $(A+)$. Les insectes apprennent d'abord cette série de discriminations binaires, puis leur connaissance de la position des items dans la séquence est éprouvée en les confrontant à des paires de repères renforcés. Dans la boîte $A$, la présentation simultanée des items $\mathrm{A}+$ et $\mathrm{D}+$ engendre des choix au hasard, alors que celle des paires $A+B+$ ou $A+C+$ entraîne une préférence significative pour l'item $A+$ qui est normalement renforcé dans cette boîte. Dans les boîtes suivantes, la présentation des paires équivalentes $(B+C+$ et $B+D+$ dans la boîte $B$, et ainsi de suite) résulte en une préférence, bien que non significative, pour l'item D+ (Pastergue-Ruiz, 1995). Des résultats similaires sont obtenus chez des abeilles Apis mellifera. Après avoir appris une séquence de trois configurations de stimuli donnant accès à une source de nourriture, elles témoignent d'une préférence pour le dernier item de la série (Collett, Fry et Wehner, 1993).

Ces résultats démontrent alors que des insectes sont capables d'apprendre une séquence de réponses, dont la dernière seulement amène au renforcement alimentaire. En revanche, ils ne semblent pas apprendre la position des items de la séquence à l'exception de celle du dernier. «II semble qu'une signification fonctionnelle particulière serait attribuée au repère le plus proche du nid. Les repères visuels de la séquence ne seraient donc pas tous mémorisés avec la même importance, ce qui rend compte d'une mémoire différentielle (en fonction des éléments de la séquence) 》 (Pastergue-Ruiz, 1995, p. 141 ; Collett et al., 1993). En d'autres termes, le renforcement obtenu lors des premiers choix corrects, correspondant au passage à la boîte suivante, diffère de celui présenté lors du dernier choix correct, l'accès au nid (fourmi) ou à la nourriture (abeille). Un lien privilégié s'établirait entre ce dernier et le repère visuel qui le précède directement.

La plupart des travaux rapportés ici ne s'intéressent qu'à une seule espèce animale, ce qui suscite plusieurs commentaires. Il faut d'abord souligner que les capacités perceptives mises en jeu lors de la résolution d'un apprentissage sont souvent spécifiques d'une espèce animale ou d'un ordre donné. Ainsi, les capacités de repérage spatial qui sont bien développées chez de nombreuses espèces vertébrées et invertébrées reposent sur des mécanismes qui different sensiblement. Par exemple, les rongeurs s'orientent dans l'espace grâce à des repères olfactifs, visuels, voire kinesthésiques tandis que les oiseaux utilisent des repères visuels et, notamment, célestes. La transposition à un cadre expérimental étant plus facile dans le premier cas, on comprend aisément que l'apprentissage de repères spatiaux soit généralement étudié chez des rongeurs. De même, en dépit de capacités 
visuelles très développées chez diverses espèces comme l'oiseau, le singe ou encore l'homme, l'organisation anatomique et physiologique du système visuel diffère de manière importante entre oiseaux et mammifères. Les espèces se distinguent également par des particularités d'ordre comportemental. Ainsi, les oiseaux témoignent d'une tendance naturelle à répéter des réponses précédemment renforcées, alors que les rongeurs explorent davantage les items non encore visités (Staddon et Simmelhag, 1971 ; Macphail et Rilley, 1989). De même, le mode d'émission des réponses diffêre entre espèces : appui sur un levier chez les rongeurs et picorage de cibles chez l'oiseau. Les comparaisons interspécifiques sont alors parfois difficiles à réaliser en raison des différences méthodologiques qui sont nécessaires pour adapter un protocole expérimental aux spécificités anatomiques et fonctionnelles d'une espèce.

Malgré ces limites méthodologiques, des similitudes ont été mises à jour entre les mécanismes d'apprentissage sériel de certaines espèces, même éloignées. Il est clairement établi que l'item occupant la dernière position d'une série possède un statut privilégié chez les vertébrés aussi bien que chez les invertébrés étudiés (Straub et Terrace, 1981 ; Mélan et al., 1996 ; Pastergue-Ruiz, 1995 ; Collett et al., 1993). Au-delà de l'apprentissage sériel, des apprentissages basés sur des mécanismes associatifs ont été décrits à travers l'ensemble du règne animal, depuis l'unicellulaire jusqu'à l'homme. Selon certains auteurs, les différences d'apprentissage rapportées dans des études comparées refléteraient alors des différences dans l'efficacité du traitement des informations entre espèces (Macphail, 1982). Selon cette conception continuiste de l'évolution phylogénétique des capacités intellectuelles, il s'agirait de différences de degré et non de nature, du moins en ce qui concerne les vertébrés (Macphail, 1985).

Les adeptes de la position discontinuiste postulent, à l'opposé, que les différences sont d'ordre qualitatif. La supériorité des capacités d'apprentissage de certaines espèces de vertébrés refléterait l'existence d'attentes qui assurent une flexibilité comportementale. Selon Bitterman (1975), une coupure se serait produite au cours de l'évolution des processus intellectuels entre les vertébrés inférieurs, poissons et reptiles, d'une part, et les vertébrés supérieurs, mammifères et oiseaux, d'autre part. D'autres auteurs, notamment De Lillo (1996), situent cette coupure entre les singes et l'homme, d'un côté, et les autres vertébrés, de l'autre côté. L'apprentissage sériel simultané révélerait des divergences fondamentales du traitement des informations sérielles chez le pigeon et le singe, dues en particulier à une différence d'empan mnésique (D'Amato et Colombo, 1988 ; Terrace, 1991 ; Swartz et al., 1991). La comparaison des performances de sujets humains et de singes lors de l'apprentissage d'une série de cinq items suggère, en revanche, l'implication de mécanismes d'apprentissage sériel similaires chez ces espèces (Stromer et Mackay, 1993). Ce débat est loin d'aboutir à un consensus, mais les travaux encouragent l'utilisation des apprentissages sériels comme un des outils d'étude de la cognition comparée. 


\section{CONCLUSION}

Dans le domaine d'étude des mécanismes d'apprentissage, un grand pas en avant pourrait être fait si les psychologues s'accordaient pour appliquer le principe de la continuité biologique entre espèces, simplement en tant qu'outil de travail. Des concepts basés sur l'expérience humaine permettraient alors de caractériser le comportement d'autres espèces et les formulations théoriques pourraient se faire à partir d'une terminologie commune (Honig, 1978). Évidemment, les conclusions issues d'une comparaison entre l'homme et d'autres espèces doivent rester prudentes en raison des capacités - en particulier de symbolisation - propres à l'homme. Ainsi, dans un apprentissage sériel, les sujets humains peuvent nommer et représenter sous forme verbale les items à mémoriser. De plus, contrairement aux animaux qui sont généralement naïfs dans ces études, ils ont l'expérience quotidienne de mémoriser des séries de codes d'appareils, de numéros de téléphone...

Les travaux résumés ici montrent que l'animal, non pourvu de capacités symboliques, apprend l'ordre de plusieurs items par étapes. Il apprend d'abord qu'il doit fournir plusieurs réponses pour être récompensé, il identifie ensuite les items qui se suivent, pour aboutir finalement à une « vue d'ensemble » de la tâche (Roitblat, 1987). Les mécanismes sous-jacents à l'apprentissage de séries semblent communs aux différentes espèces de vertébrés étudiées. À l'appui de cette hypothèse, rappelons le fameux principe de parcimonie de Lloyd Morgan : « Dans aucun cas nous ne pouvons interpréter une action comme le résultat de la mise en jeu d'une fonction psychologique supérieure, si elle peut être interprétée comme le résultat de la mise en jeu d'une fonction située plus bas dans l' "échelle" psychologique " (1894, dans Macphail, 1982, p. 1) ${ }^{1}$. Rats, pigeons et singes sont capables de transférer des règles apprises en présence de stimuli particuliers à de nouveaux stimuli (Hulse et Dorsky, 1979 ; Terrace, 1986 b ; Roitblat, 1987 ; Swartz et al., 1991 ; Terrace et al., 1995 ; Mélan et al., 1998). L'idée centrale des théories associatives classiques, qui suggèrent une association particulière entre un stimulus et une réponse donnés, s'avère alors trop restrictive pour rendre compte de l'ensemble de ces données. Les résultats indiquent que l'animal traite activement des informations qu'il mémorise ensuite sous une forme qui lui permet de les appliquer à d'autres situations. La représentation des informations par une image mentale résulterait de la mise en jeu de processus dits plus élémentaires.

Les travaux montrent à l'unanimité que l'animal identifie et mémorise le mieux l'item occupant la dernière position d'une série, et à un moindre

1. In no case may we interpret an action as the outcome of the exercise of a higher psychical faculty, if it can be interpreted as the outcome of the exercise of one which stands lower in the psychological scale. 
degré, celui occupant la première position. Ceci explique que les performances dépendent directement de l'intervalle de rétention utilisé ainsi que du nombre d'items composant une série. L'apprentissage de règles relatives à ces items, plus faciles à discriminer, sont suffisantes pour expliquer l'apprentissage d'une série de deux items. À partir de séries de trois items l'animal semble relier les items entre eux pour former une série plutôt que d'apprendre des règles supplémentaires (Straub et Terrace, 1981 ; Roitblat, 1987 ; D'Amato, 1991 ; Castéras et al., 1997). Parmi les informations sur lesquelles l'animal se base pour organiser ses réponses multiples en une séquence ordonnée, citons les manifestations proprioceptives corrélatives des mouvements participant à l'émission d'une réponse (D'Amato, 1991), l'intervalle de temps qui sépare l'émission d'une réponse de la distribution du renforcement appétitif (D'Amato, 1991 ; Castéras et al., 1997) ou encore les représentations internes des stimuli ou des réponses au stimuli (Roitblat, 1987 ; D’Amato, 1991 ; Terrace, 1993). Tout laisse penser que le singe développe à partir de ces informations une représentation linéaire des items d'une série, même longue. L'empan mnésique étant plus limité chez le pigeon, celui-ci formerait une représentation de nature associative d'une série longue. Lorsque les conditions expérimentales favorisent le regroupement d'items en unités fonctionnelles, celles-ci seraient représentées de manière plus élaborée, même chez le pigeon.

\section{RESUMÉ}

Les mécanismes d'apprentissage sériel sont étudiés chez les vertébrés supérieurs grâce à deux types de protocoles. Dans l'un, l'animal doit reproduire l'ordre de présentation de plusieurs éléments présentés successivement, sollicitant fortement la mémoire à court terme. Dans l'autre, l'animal découvre l'ordre dans lequel il doit répondre à des éléments présentés simultanément. Les mécanismes d'apprentissage sériel incluent des processus discriminatifs, l'apprentissage de règles et le développement d'une représentation mentale. Ils ne s'excluent pas mutuellement pour un apprentissage donné, mais semblent varier selon l'espèce étudiée, et divers paramètres expérimentaux.

Mots-clés : apprentissage sériel, processus de discrimination, représentation mentale, comparaison interspécifique.

\section{BIBLIOGRAPHIE}

Bever T. G. - (1984) The road from behaviorism to rationalism, in H. L. Roitblat, T. G. Bever et H. S. Terrace (Edit.), Animal cognition, Hillsdale (NJ), Erlbaum, 61-75.

Bitterman M. E. - (1975) The comparative analysis of learning, Science, 188, 699-709.

Bloom J. M., Capaldi E. J. - (1961) The behavior of rats in relation to complex patterns of partial reinforcement, Journal of Comparative and Physiological Psychology, 54, 261-265. 
Camps J.-F. - (1998) Étude comparée d'apprentissage de séries chez l'humain et chez le pigeon : approche comportementale et modelisation, thèse de doctorat en Neurosciences, Université Toulouse 3.

Camps J.-F., Castéras V., Mélan C., Delius J. - (1998) Studies of simultaneous 3-item series acquisition in pigeons, European Journal of Neuroscience, Supplement $10,153$.

Camps J.-F., Durif C., Mélan C., Delius J. - (1998) Learning of a two-item series in a free correction procedure, in $\mathbf{N}$. Elsner et $R$. Wehner (Edit.), New neuroethology on the move, Stuttgart, Thieme, 529.

Camps J.-F., Mélan C. - (1999) De l'organisation sérielle des comportements à l'apprentissage sériel, in J. Gervet et M. Pratte, Éléments d'éthologie cognitive, Paris, Hermes Science Publications, 221-240.

Castéras V. - (1996) Étude des processus impliqués dans l'acquisition de tâches complexes chez le pigeon (Columba livia), thèse de doctorat en Neurosciences, Université Toulouse 3.

Castéras V., Camps J.-F., Mélan C. - (1997) Serial learning in the pigeon in a free correction procedure : Role of reinforcing values, Comptes rendus de l'Académie des Sciences, 320, 375-384.

Capaldi E. J., Verry D. R. - (1981) Serial order anticipation learning in rats : Memory for multiple hedonic events and their order, Animal Learning and Behavior, 9, 441-453.

Capaldi E. J., Verry D. R., Davidson T. L. - (1980) Memory, serial anticipation pattern learning and transfer in rats, Animal Learning and Behavior, 8 , 575-585.

Collett T. S., Fry S. N., Wehner R. - (1993) Sequence learning by honeybees, Journal of Comparative Physiology, 172, 693-706.

Compton D. M. - (1991) Serial learning ; a review of behavioral and physiological research with the rat, Neuroscience and Biobehavioral Reviews, 15, 363 374.

D'Amato M. R. - (1991) Comparative cognition : Processing of serial order and serial pattern, in L. Dachowsky et C. F. Flatherty (Edit.), Current topics in animal learning : Brain emotion and cognition, Hillsdale (NJ), Erlbaum, 165-187.

D'Amato M. R., Colombo M. - (1988) Representation of serial order in monkeys (Cebus apella), Journal of Experimental Psychology, Animal Behavior Processes, 14, 131-139.

D'Amato M. R., Colombo M. - (1989) Serial learning with wild card items by monkeys (Cebus apella) : Implications for knowledge of ordinal position, Journal of Comparative Psychology, 103, 252-261.

De Lillo C. - (1996) The serial organisation of behaviour by non-human primates; an evaluation of experimental paradigms, Behavioural Brain Research, 91, 1-17.

Ebbinghaus H. (Edit.) - (1913) Memory : A contribution to experimental psychology, New York, Columbia University.

Guthrie E. R. (Edit.) - (1935) The psychology of learning, New York, Harper \& Row.

Honig W. K. - (1978) On the conceptual nature of cognitive terms : An initial essay, in S. H. Hulse, H. Fowler et W. K. Honig (Edit.), Cognitive processes in animal behavior, New York, Erlbaum, 1-14.

Hull C. L. - (1931) Goal attraction and directing ideas conceived as habit phenomena, Psychological Review, 38, 487-506.

Hulse S. H. - (1978) Serial pattern learning, in S. H. Hulse, H. Fowler et 
W. K. Honig (Edit.), Cognitive processes in animal behavior, New York, Erlbaum, 31 1-340.

Hulse S. H., Campbell C. E. - (1975) « Thinking ahead » in rat discrimination learning, Animal Learning and Behavior, 3, 305-311.

Hulse S. H., Dorsky N. P. - (1979) Structural complexity as a determinant of serial pattern learning, Learning and Motivation, 8, 488-506.

Hunter W. S. - (1920) The temporal maze and kinaesthetic sensory processes in the white rat, Psychobiologv, 2, 1-17.

Lashley K. S. - (1951) The problem of serial order in behavior, in L. A. Jeffress (Edit.), Cerebral mechanisms in behavior, New York, Wiley, $112-136$.

lewandowsky S., Murdock B. B. - (1989) Memory for serial order, Psychological Review, 96, 25-57.

Macdonald S. E. - (1993) Delayed matching-to-successive samples in pigeons : Short-term memory for item and order information, Animal Learning and Behavior, I, 59-67.

Macphail E. M. (Edit.) - (1982) Brain and intelligence in vertebrates, Oxford, Clarens Press.

Macphail E. M. - (1985) Vertebrate intelligence: The null hypothesis, in I. Weiskrantz (Edit.), Animal intelligence, New York, Oxford University Press, 37-51.

Macphail E. M., Reilly S. - (1989) Rapid acquisition of novelty versus familiarity concept by pigeons (Columba livia), Journal of Experimental Psychology, Animal Behavior Processes, 15, 242-252.

Mélan C., Castéras V., Corbière M., Gallo A. - (1996) Response strategies during acquisition of serial learning in pigeons (Columba livia), Behavioural Processes, 36, 39-50.

Mélan C., Galy E., Camps J.-F. - (1998) Human and pigeon serial learning generalisation in a free correction procedure, European Journal of Neuroscience, Supplement 10, 211.

Mélan C., Ungerer A. - (1991) Positional choices and visual discrimination in non-delayed appetitive tasks in mice, Behavioural Processes, 23, 137 143.

Olton D. S., Samuelson R. J. - (1976) Remembrance of places passed : Spatial memory in rats, Journal of Experimental Psychology, Animal Behavior Processes, 2, 97-116.

Pastergue-Ruiz I. - (1995) La représentation visuelle de l'espace chez un insecte : la fourmi Cataglyphis cursor, thèse de doctorat en Neurosciences, Université Toulouse 3.

Roitblat H. L. (Edit.) - (1987) Introduction to comparative cognition, New York, W. H. Freeman \& Co.

Shimp C. P. - (1976) Short-term memory in the pigeon : Relative recency, Journal of Experimental Analysis of Behavior, 25, 55-61.

Skinner B. F. (Edit.) - (1938) The behavior of organisms, New York, AppletonCentury-Crofts.

Staddon J. E. R., Simmelhag V. L. - (1971) The superstition experiment : A reexamination of its implications for the principles of adaptative behavior, Psychological Review, 78, 3-43.

Straub R. O., Seidenberg M. S., Bever T. G., Terrace H. S. - (1979) Serial learning in the pigeon, Journal of Experimental Analysis of Behavior, 32, 137148.

Straub R. O., Terrace H. S. - (1981) Generalisation of serial learning in the pigeon, Animal Learning and Behavior, 9, 454-468. 
Stromer R., Mackay H. A. - (1993) Delayed identity matching to complex samples : Teaching students with mental retardation spelling and the prerequisites for equivalence classes, Research Revue Disabilities, 14, 19-38.

Swartz K. B., Chen S., Terrace H. S. - (1991) Serial learning by rhesus monkeys : I. Acquisition and retention of multiple four-item lists, Journal of Experimental Psychology, Animal Behavior Processes, 17, 396-410.

Terrace H. S. - (1986 a) Positive transfer from sequence production to sequence discrimination in a nonverbal organism, Journal of Experimental Psychology, Animal Behavior Processes, 12, 215-234.

Terrace H. S. - (1986 b) A nonverbal organism's knowledge of ordinal position in a serial learning task, Journal of Experimental Psychology, Animal Behavior Processes, 12, 203-214.

Terrace H. S. - (1987) Chunking by a pigeon in serial learning task, Nature, $325,149-151$.

Terrace H. S. - (1991) Chunking during serial learning by a pigeon : Basic evidence, Journal of Experimental Psychology, Animal Behavior Processes, 17 , 81-93.

Terrace H. S. - (1993) The phylogeny and ontogeny of serial memory : List learning by pigeons and monkeys, Psychological Science, 4, 162-169.

Terrace H. S., Chen S., Jaswal V. - (1996) Recall of three-item sequences by pigeons, Animal Learning and Behavior, 24, 193-205.

Terrace H. S., Chen S., Newman A. B. - (1995) Serial learning with a wild card by pigeons (Columba livia) : Effect of list length, Journal of Comparative Psychology, 109, 162-172.

Thorndike E. L. (Edit.) - (1913) Educational Psychology : II The psychology of learning, New York, Teachers' College.

Tolman E. C. - (1948) Cognitive maps in rats and man, Psychological Review, $55,189-208$.

Wasserman E. A., Nelson K. R., Larew M. B. - (1980) Memory for sequences of stimuli and responses, Journal of the Experimental Analysis of Behavior, 48, 573-607.

Watson J. B. - (1913) Psychology as the behaviorist views it. Psychological Review, 20, 158-177.

Weisman R. G., Wasserman E. A., Nelson K. R., Larew M. B. - (1980) Representation and retention of two-event sequences in pigeons, Journal of Experimental Psychology, Animal Behavior Processes, 6, 312-325. 